\title{
Coronary revascularization after surgical aortic valve replacement
}

Mevlüt Çelik, BSc, ${ }^{a}$ Andras P. Durko, MD, ${ }^{\text {a }}$ Stuart J. Head, MD, PhD, ${ }^{\text {a,b }}$ Edris A. F. Mahtab, MD, PhD, ${ }^{a}$ Nicolas M. van Mieghem, MD, PhD, ${ }^{c}$ Paul A. Cummins, MSc, ${ }^{c}$ Arie P. Kappetein, MD, PhD, ${ }^{a, b}$ and Ad. J. J. C. Bogers, MD, PhD

\section{ABSTRACT}

Objective: It remains unclear how often coronary revascularization is necessary after aortic valve interventions, either by surgical aortic valve replacement (SAVR) or transcatheter aortic valve replacement. However, these data are relevant for treatment and prosthesis choice. The authors sought to analyze the incidence and characteristics of coronary revascularization after SAVR during follow-up.

Methods: Of 2256 patients undergoing isolated SAVR between 1987 and 2015, 420 patients (mean age $56.9 \pm 15.5$ years, $66.9 \%$ male) were followed at the Erasmus Medical Center. Incidence, predictors, and characteristics of coronary revascularization were analyzed. Cumulative incidence of revascularization was assessed using a competing risk approach.

Results: Mean follow-up after SAVR was 17.2 years (total of 4541 patient-years). A total of 24 patients underwent 28 procedures of revascularization. The cumulative incidence of revascularization after SAVR was $0.5 \%, 2.2 \%, 4.1 \%$, and $6.9 \%$ at 1,5 , 10 , and 20 years, respectively. The linearized rate of revascularization was 6.2 per 1000 patient-years. Percutaneous coronary intervention was the most common revascularization method $(64 \% ; N=18 / 28)$. Revascularization before SAVR ( $N=36 / 420$; of whom 27 percutaneous coronary intervention) was an independent predictor of revascularization during follow-up (hazard ratio, 6.6; 95\% confidence interval, 2.6-17.1; $P<$.001).

Conclusions: After SAVR, the rate of coronary revascularization was $6.9 \%(\mathrm{~N}=24)$ 420) at 20-year follow-up. Patients were at particular risk if they had undergone previous revascularization before SAVR. These data may furthermore be relevant to the transcatheter aortic valve replacement population. (JTCVS Open 2020;3:91-101)

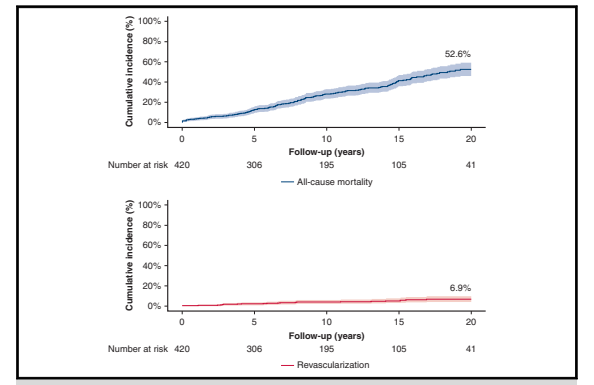

Competing risk cumulative incidences of mortality and revascularization; 20-year follow-up.

CENTRAL MESSAGE

In a large SAVR cohort, the rate of coronary revascularization was $6.9 \%$ after 20-year follow-

up. Previous revascularization was an independent predictor of revascularization after SAVR during follow-up.

\section{PERSPECTIVE}

Coronary revascularization rates after SAVR can be used to predict the need for revascularization after TAVR, should TAVR further expand into younger, lower-risk populations. Dedicated studies are required to address the incidence, predictors, and feasibility of revascularization after TAVR.

See Commentaries on pages 102 and 104 .
From the Departments of ${ }^{\mathrm{a}}$ Cardiothoracic Surgery and ${ }^{\mathrm{c}}$ Cardiology, Erasmus University Medical Center, Rotterdam; and ${ }^{\mathrm{b}}$ Medtronic, Maastricht, The Netherlands.

Received for publication May 8, 2020; revisions received May 8, 2020; accepted for publication May 8, 2020; available ahead of print July 17, 2020.

Address for reprints: Edris A. F. Mahtab, MD, PhD, Department of Cardiothoracic Surgery, Erasmus MC, Rotterdam, The Netherlands (E-mail: E.Mahtab@ erasmusmc.nl).

2666-2736

Copyright (C) 2020 The Authors. Published by Elsevier Inc. on behalf of The American Association for Thoracic Surgery. This is an open access article under the CC BY license (http://creativecommons.org/licenses/by/4.0/).

https://doi.org/10.1016/j.xjon.2020.05.005
Transcatheter aortic valve replacement (TAVR) is now recommended for patients with severe aortic valve stenosis (AS) at intermediate and high surgical risk, ${ }^{1,2}$ adding more evidence to the already ongoing increase in the number of performed TAVR procedures in North America and Europe. ${ }^{3,4}$ Recent trials that included low-risk patients have reported noninferiority or even superiority of TAVR versus surgical aortic valve replacement (SAVR). ${ }^{5,6}$ 


\section{Abbreviations and Acronyms \\ AS = aortic valve stenosis \\ $\mathrm{CABG}=$ coronary artery bypass grafting \\ $\mathrm{CAD}=$ coronary artery disease \\ $\mathrm{LVEF}=$ left ventricular ejection fraction \\ PCI = percutaneous coronary intervention \\ SAVR $=$ surgical aortic valve replacement \\ TAVR $=$ transcatheter aortic valve replacemen}

Reports have suggested that access to the coronary arteries may be difficult to establish after TAVR as a result of the positioning of the transcatheter valve. ${ }^{7}$ When indication expands toward low-risk patients, who often are younger, the need for coronary revascularization after TAVR may increase. However, due to the advanced age and presence of multiple comorbidities of patients in current TAVR trials and the relatively short-term follow-up available, the incidence of coronary revascularization has been difficult to determine. The probability of coronary revascularization after TAVR may increase in patients with longer life expectancies, with potential implications for procedure and prosthesis choices.

SAVR has been the standard of care for AS over the past 50 years. Therefore, long-term follow-up is available to determine the incidence of coronary revascularization after SAVR in low-risk patients. Since the historical SAVR patient population overlaps with current and future TAVR patient populations, data of revascularization after SAVR can provide insights into determining which surgical or transcatheter prostheses may be more appropriate in specific patients. The aim of this study was to assess the incidence and risk factors of coronary revascularization during long-term follow-up after SAVR.

\section{METHODS \\ Study Design}

This observational, retrospective study consisted of adult ( $\geq 18$ years) patients who underwent isolated SAVR with a mechanical or bioprosthetic valve between 1987 and 2015 at the Erasmus Medical Center (Erasmus MC), Rotterdam, The Netherlands. To ensure that all coronary revascularization procedures during follow-up were captured, only patients followed up at the outpatient clinic of the Erasmus MC were included in this study (Figure 1). Patients undergoing concomitant procedures or with active endocarditis were excluded. Coronary artery disease (CAD) was routinely assessed before SAVR by coronary angiography, and patients with CAD underwent concomitant coronary artery bypass grafting (CABG) according to the recommendations of clinical guidelines in use at the time of surgery and were excluded.

The study was approved by the local institutional review board, and patient-informed consent was waived. All the authors assured for the validity of the data and adherence to the protocol.

\section{Data Collection and Follow-up}

Baseline patient and procedural characteristics were collected from electronic medical records. Survival status was obtained through the National Death Registry.
After SAVR, patients returned to their referring cardiologist at Erasmus MC for routine, regular outpatient clinic visits at 3 and 6 months postoperatively and (bi-)annually thereafter. If CAD was diagnosed and revascularization was deemed necessary, patients underwent either percutaneous coronary intervention (PCI) or CABG at the Erasmus MC.

\section{End Points and Definitions}

The primary end point was coronary revascularization either by $\mathrm{PCI}$ or CABG. SAVR within 24 hours of establishing the indication was classified as urgent, between 24 hours and 3 days as semi-elective, and after 3 days as elective. Left ventricular function was classified as normal if the left ventricular ejection fraction (LVEF) was $>50 \%$, as mildly reduced if the LVEF was $40 \%$ to $50 \%$, as moderately reduced if the LVEF was $30 \%$ to $40 \%$, and as severely reduced if the LVEF was less than $30 \%$, as measured or estimated by a trained echocardiographer.

\section{Statistical Analyses}

Discrete variables are presented as numbers, percentages or proportions, and compared with either the $\chi^{2}$ test or the Fisher exact test, where appropriate. Continuous variables are presented as means \pm standard deviation or median with the interquartile range if there was evidence of skewed data according to the Kolmogorov-Smirnov test, and compared with either the 2-sample $t$ test or Wilcoxon rank-sum test, where appropriate.

Probabilities of the occurrence of revascularization and mortality were visualized using cumulative incidence curves with their according $95 \%$ confidence intervals. The cumulative incidence based on Kaplan-Meier estimates does not reflect the competing risk of death and the occurrence of revascularization and therefore overestimate the remaining lifetime risk of revascularization when the competing risk is high. ${ }^{8}$ To account for this overestimation, competing risk survival analysis was performed by means of nonparametric methods using the cumulative incidence competing risk method. ${ }^{9,10}$ Post-hoc subgroup analyses were performed according to whether revascularization had taken place before the SAVR procedure, age at time of SAVR (aged $<65$ or $\geq 65$ years), history of hypercholesterolemia, history of diabetes mellitus, indication of SAVR (AS, aortic valve regurgitation, or combined disease), and type of implanted valve (mechanical or bioprosthetic). Competing risk survival analyses in subgroups were compared with the Fine and Gray test. ${ }^{11}$ Furthermore, the linearized rate of revascularization was calculated per 1000 patient-years of follow-up.

Predictors of revascularization after SAVR were identified in a Cox proportional hazards model. Significant variables on univariable analyses were included in a multivariable Cox proportional hazards model. Data analyses were performed using SPSS 24.0 (IBM Corp, Armonk, NY) and R software, version 3.4 (R Foundation for Statistical Computing, Vienna, Austria).

\section{RESULTS}

\section{Baseline and Procedural Characteristics}

From 4228 patients who underwent SAVR between 1987 and 2015, 420 patients underwent isolated SAVR and were followed up at the Erasmus MC and were included in this study (Figure 1). The mean age of the patients at the time of SAVR was $56.9 \pm 15.5$ years, and $66.9 \%$ (281/420) were male. The primary indication for SAVR was pure AS in $52.1 \%$ (219/420). A total of 8.6\% (36/420) had previous revascularization. Mechanical valve prostheses were used in $66.7 \%(280 / 420)$. The rates of survival were $98.3 \%, 96.4 \%, 87.4 \%, 71.8 \%, 58.6 \%$, and $47.4 \%$ at 30 days, and 1, 5, 10, 15, and 20 years of follow-up, respectively (Figure 2). Detailed baseline and procedural 


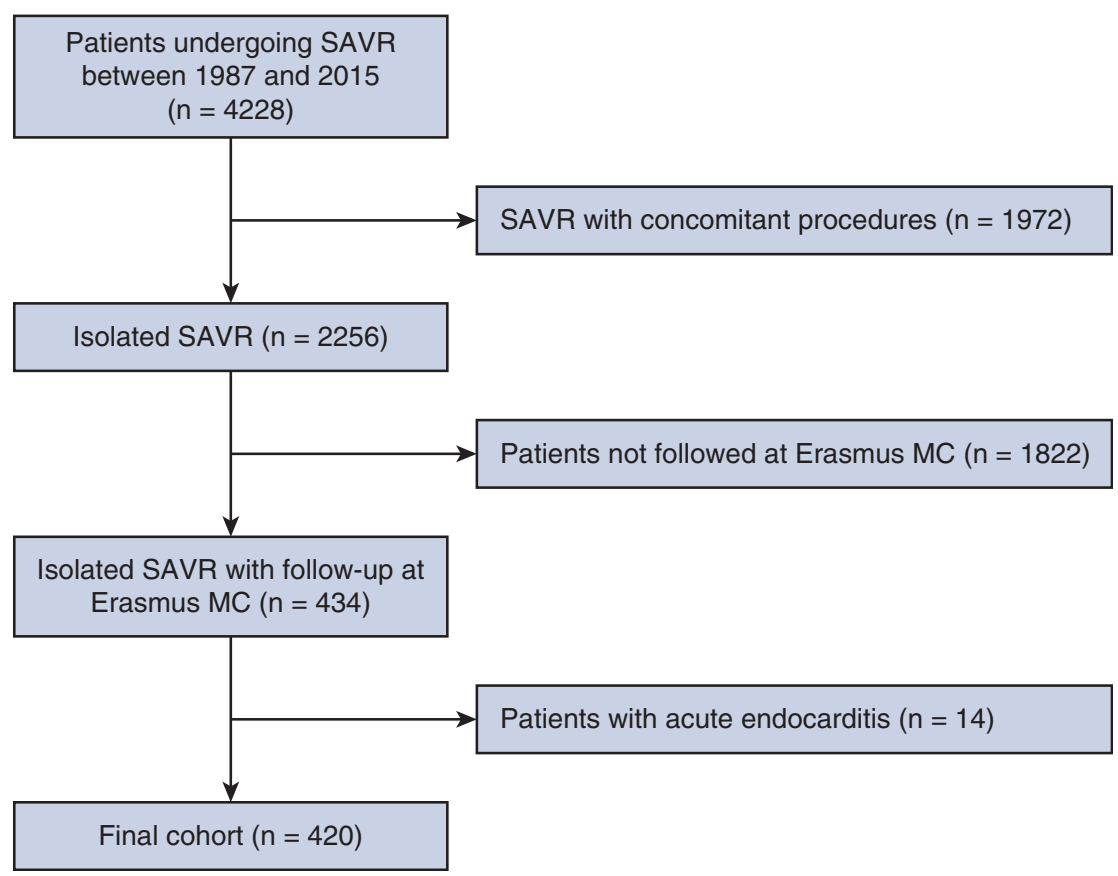

FIGURE 1. Flowchart of patient inclusion. A total of 4228 patients underwent SAVR at the Erasmus MC between 1987 and 2015 , of whom a total of 420 patients were eligible for the study. SAVR, Surgical aortic valve replacement.

characteristics are provided in Table 1. Patients excluded from our study were older $(66.1 \pm 11.1$ vs $56.9 \pm 15.5$ years, $P<.001$ ), had undergone more redo SAVR procedures $(16.7 \%$ vs $4.3 \%, P<.001)$, more often underwent SAVR with an urgent indication $(4.0 \%$ vs $0.4 \%$, $P<.001$ ), and had less-frequent implantation of mechanical valve prosthesis $(66.7 \%$ vs $48.0 \% P<.001)$ compared with the included patients. Further detailed characteristics of patients excluded from our study are provided in Table 2.

\section{Revascularization After SAVR}

The mean follow-up after SAVR was 17.2 years, with a total follow-up accumulating to 4541 patient-years. During follow-up, 24 patients underwent coronary revascularization, with 3 patients requiring a second and 1 patient requiring a third revascularization procedure. In the timeto-first event competing risk analysis with mortality, the rates of revascularization were $0.5 \%, 0.5 \%, 2.2 \%, 4.1 \%$, $5.3 \%$, and $6.9 \%$ at 30 days and $1,5,10,15$, and 20 years of follow-up, respectively (Figure 2). The mean time to the first revascularization was $8.9 \pm 7.4$ (range 026.9 years). The linearized rate of revascularization was 6.2 per 1000 patient-years.

\section{Characteristics of Revascularization}

More patients underwent $\mathrm{PCI}$ than $\mathrm{CABG}$, accounting for $64.2 \%$ of revascularization procedures $(n=18 / 28)$. Three patients $(12.5 \%)$ needed urgent revascularization due to acute myocardial infarction (treated with PCI in all cases). Single-vessel disease was present in 16 patients $(67 \%)$ and multivessel disease was present in 8 patients $(33 \%)$. Four patients had lesions in both the left and right coronary artery. Characteristics of revascularization are displayed in Table 3.

\section{Subgroup Analysis and Predictors of Revascularization After SAVR}

The incidence of revascularization at 15 years of followup was significantly greater in patients with previous revascularization than in patients without previous revascularization $(22.1 \%$ vs $3.7 \%, P<.001)$, respectively. Further, the incidence of revascularization was greater in patients with hypercholesterolemia compared with patients without hypercholesterolemia $(14.2 \%$ vs $4.1 \%, P=.002)$, respectively. There were no differences in revascularization rates during follow-up in subgroups according to age $(4.9 \%$ for patients aged $<65$ vs $5.9 \%$ for patients aged $\geq 65$, $P=.42)$, diabetes mellitus $(8.8 \%$ for patients with a history of diabetes mellitus vs $5.0 \%$ for no diabetes mellitus, $P=.24)$, primary indication for SAVR $(5.6 \%$ for AS vs $7.9 \%$ for aortic valve regurgitation vs $2.2 \%$ for combined disease, $P=.36)$, or type of valve used $(6.8 \%$ for biological vs $4.4 \%$ for mechanical, $P=.16$ ) (Figures 3 and 4 ).

\section{Factors Associated With Coronary \\ Revascularization During Follow-up}

Patients who underwent coronary revascularization during follow-up more often had hypercholesterolemia at baseline ( $8 / 24$ vs 44/396, $P=.001)$ and undergone revascularization before the index procedure (7/24 vs $29 / 396$, 

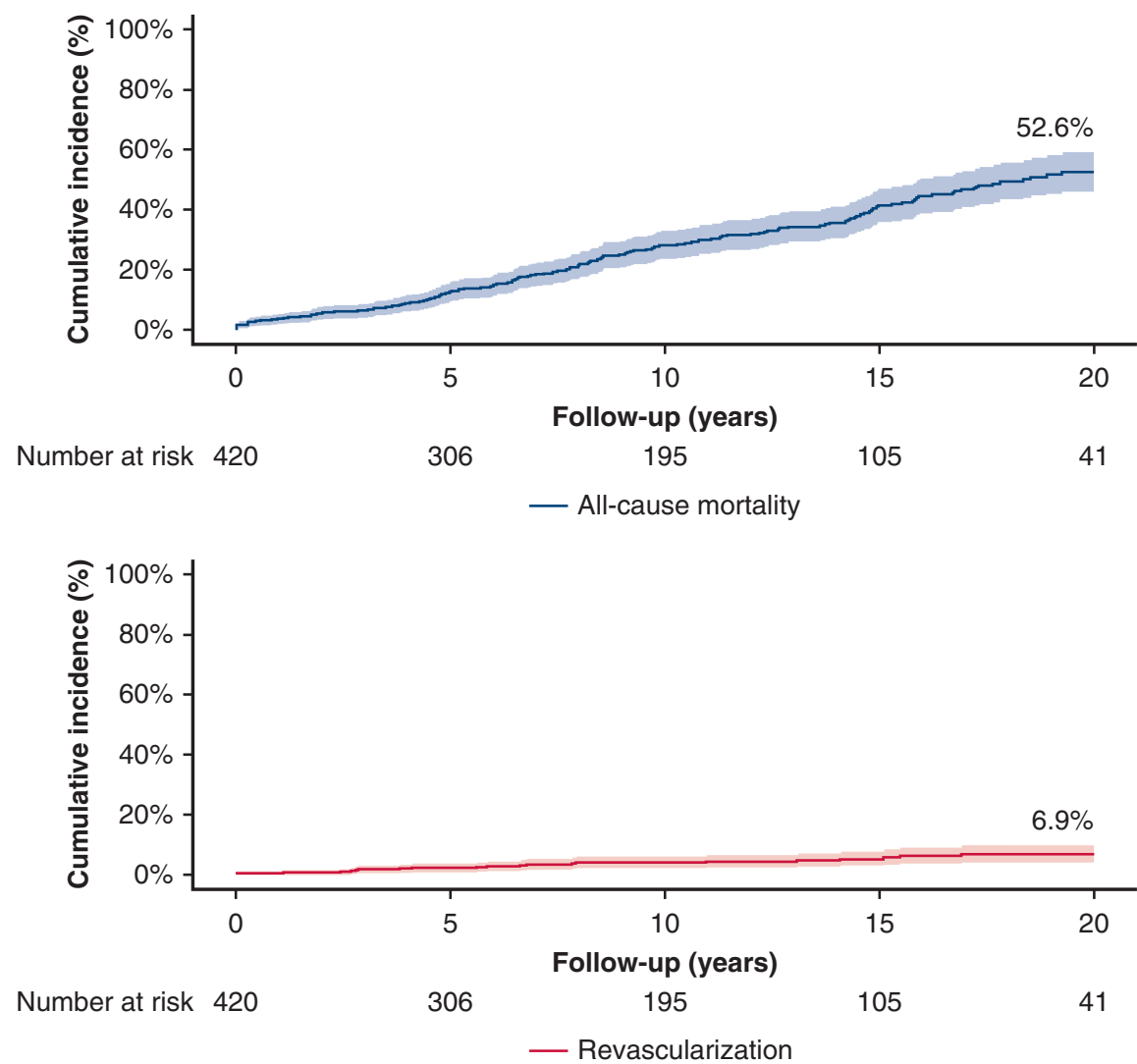

FIGURE 2. Mortality and coronary revascularization after SAVR. Competing risk cumulative incidences of mortality and coronary revascularization during 20-year follow-up according to (A) blue line presents the cumulative incidence of all-cause mortality competing with the risk of revascularization in our cohort and (B) red line presents the cumulative incidence of revascularization with either PCI or CABG competing with the risk of revascularization in our cohort. SAVR, Surgical aortic valve replacement; $P C I$, percutaneous coronary intervention; $C A B G$, coronary artery bypass grafting.

$P<.001)$ than patients that did not undergo revascularization during follow-up (Table 1). In multivariable analyses, the presence of revascularization, hypercholesterolemia, and diabetes mellitus before the index procedure were the only independent predictor of revascularization during follow-up (Table 4).

\section{DISCUSSION}

In this cohort of 420 patients who underwent isolated SAVR, $24(5.7 \%)$ patients underwent a total of 28 revascularization procedures. The cumulative incidence of revascularization was $6.9 \%$ at 20 -year follow-up, with a linearized rate of 6.2 per 1000 patient-years. In the current study, concomitant CABG was generally performed in patients with significant coronary stenosis. The risk of requiring coronary intervention during follow-up after SAVR in patients with no significant coronary stenosis at the time of intervention appears to be low as $6.9 \%$ at 20-year follow-up (Figure 5).

The incidence of revascularization was greater than that of the general population. Subgroup analyses showed that patients who had undergone previous revascularization before SAVR and patients with a history of hypercholesterolemia had significantly greater rates of revascularization during follow-up. Clearly patients with already established CAD, but nonsignificant at the time of SAVR, carry a risk of progression of CAD to a severity requiring intervention. Other risk factors of $\mathrm{CAD}$, like hypertension and diabetes, were not associated with revascularization in our multivariable analysis, although this may be the result of a relatively low sample size in our study.

Of the patients who underwent revascularization, 16 patients had single-vessel disease and 8 patients 2 -vessel disease. There were no patients with left main or 3-vessel disease. Considering the current guidelines for revascularization, the majority of patients would be referred for PCI on the basis of the complexity of coronary disease. ${ }^{12}$ Eight patients with more complex coronary disease underwent CABG during follow-up.

These data are important in the era of expanding indications for TAVR. Recently, 2 randomized controlled trials showed significant benefit of TAVR compared with SAVR in the low-risk population. ${ }^{5,6}$ Revascularization with PCI after TAVR can be associated with multiple technical challenges related to transcatheter heart valve 
TABLE 1. Baseline and procedural characteristics

\begin{tabular}{|c|c|c|c|c|}
\hline & All patients $(n=420)$ & No revascularization $(n=396)$ & Revascularization $(n=24)$ & $P$ value \\
\hline Age, y & $56.9 \pm 15.5(420)$ & $56.8 \pm 15.7(396)$ & $58.5 \pm 11.6(24)$ & .592 \\
\hline Male sex & $66.9(281 / 420)$ & $67.2(266 / 396)$ & $62.5(15 / 24)$ & .637 \\
\hline $\begin{array}{l}\text { Primary indication } \\
\text { AS } \\
\text { AR } \\
\text { Combined AS + AR }\end{array}$ & $\begin{array}{l}52.1(219 / 420) \\
25.5(107 / 420) \\
22.4(94 / 420)\end{array}$ & $\begin{array}{l}52.3(207 / 396) \\
25.5(101 / 396) \\
22.2(88 / 396)\end{array}$ & $\begin{array}{l}50.0(12 / 24) \\
25.0(6 / 24) \\
25.0(6 / 24)\end{array}$ & .950 \\
\hline Bicuspid aortic valve & $24.0(101 / 420)$ & $24.0(95 / 396)$ & $25.0(6 / 24)$ & .910 \\
\hline $\begin{array}{l}\text { Previous cardiac operation } \\
\text { SAVR } \\
\text { CABG } \\
\text { Other }\end{array}$ & $\begin{array}{c}28.6(120 / 420) \\
16.7(70 / 420) \\
2.6(11 / 420) \\
9.3(39 / 420)\end{array}$ & $\begin{array}{c}28.8(114 / 396) \\
16.7(66 / 396) \\
2.3(9 / 396) \\
9.3(39 / 396)\end{array}$ & $\begin{array}{c}25.0(6 / 24) \\
16.7(4 / 24) \\
8.3(2 / 24) \\
0\end{array}$ & $\begin{array}{r}.690 \\
>.999 \\
.071 \\
.107\end{array}$ \\
\hline Hypertension & $29.8(125 / 420)$ & $29.8(118 / 396)$ & $29.2(7 / 24)$ & .948 \\
\hline Hypercholesterolemia & $12.4(52 / 420)$ & $11.1(44 / 396)$ & $33.3(8 / 24)$ & .001 \\
\hline Diabetes mellitus & $9.3(39 / 420)$ & $8.8(35 / 396)$ & $16.7(4 / 24)$ & .199 \\
\hline $\begin{array}{l}\text { Arterial disease } \\
\text { Peripheral } \\
\text { Carotid }\end{array}$ & $\begin{array}{l}3.6(15 / 420) \\
3.6(15 / 420) \\
0.5(2 / 420)\end{array}$ & $\begin{array}{l}3.3(13 / 396) \\
3.3(13 / 396) \\
0.5(2 / 396)\end{array}$ & $\begin{array}{c}8.3(2 / 24) \\
8.3(2 / 24) \\
0\end{array}$ & $\begin{array}{l}.195 \\
.195 \\
.727\end{array}$ \\
\hline Renal failure & $2.6(11 / 420)$ & $2.5(10 / 420)$ & $4.2(1 / 24)$ & .625 \\
\hline Previous myocardial infarction & $4.3(18 / 420)$ & $4.0(16 / 396)$ & $8.3(2 / 24)$ & .313 \\
\hline $\begin{array}{l}\text { Previous revascularization } \\
\text { Previous PCI } \\
\text { Previous CABG }\end{array}$ & $\begin{array}{l}8.6(36 / 420) \\
6.4(27 / 420) \\
2.6(11 / 420)\end{array}$ & $\begin{array}{l}7.3(29 / 396) \\
5.6(22 / 396) \\
2.3(9 / 396)\end{array}$ & $\begin{array}{r}29.2(7 / 24) \\
20.8(5 / 24) \\
8.3(2 / 24)\end{array}$ & $\begin{array}{r}<.001 \\
.003 \\
.071\end{array}$ \\
\hline Previous decompensated heart failure & $16.9(71 / 420)$ & $16.4(65 / 396)$ & $25.0(6 / 24)$ & .276 \\
\hline $\begin{array}{l}\text { Left ventricular function } \\
\text { Preserved } \\
\text { Mildly reduced } \\
\text { Moderately reduced } \\
\text { Severely reduced }\end{array}$ & $\begin{array}{l}77.6(287 / 370) \\
7.6(28 / 370) \\
9.2(34 / 370) \\
5.7(21 / 370)\end{array}$ & $\begin{array}{l}77.6(273 / 370) \\
8.0(28 / 370) \\
8.8(31 / 370) \\
5.7(20 / 370)\end{array}$ & $\begin{array}{c}77.8(14 / 18) \\
0 \\
16.7(3 / 18) \\
5.6(1 / 18)\end{array}$ & .460 \\
\hline Atrial fibrillation & $13.3(56 / 420)$ & $13.4(53 / 396)$ & $12.5(3 / 24)$ & .902 \\
\hline $\begin{array}{l}\text { Previous neurologic event } \\
\text { CVA } \\
\text { TIA }\end{array}$ & $\begin{array}{r}10.5(44 / 420) \\
4.8(20 / 420) \\
7.1(30 / 420)\end{array}$ & $\begin{array}{r}11.1(44 / 396) \\
5.1(20 / 396) \\
7.6(30 / 396)\end{array}$ & $\begin{array}{l}0 \\
0 \\
0\end{array}$ & $\begin{array}{l}.084 \\
.259 \\
.162\end{array}$ \\
\hline COPD & $8.3(35 / 420)$ & $8.3(33 / 396)$ & $8.3(2 / 24)$ & $>.999$ \\
\hline Liver disease & $1.4(6 / 420)$ & $1.5(6 / 396)$ & 0 & .544 \\
\hline History of malignancy & $8.1(34 / 420)$ & $8.1(32 / 396)$ & $8.3(2 / 24)$ & .965 \\
\hline $\begin{array}{l}\text { Urgency } \\
\text { Elective } \\
\text { Semi-elective } \\
\text { Urgent }\end{array}$ & $\begin{array}{c}49.3(173 / 351) \\
46.7(164 / 351) \\
4.0(14 / 351)\end{array}$ & $\begin{array}{c}49.4(165 / 334) \\
46.7(156 / 334) \\
3.9(13 / 334)\end{array}$ & $\begin{array}{r}47.1(8 / 17) \\
47.1(8 / 17) \\
5.9(1 / 17)\end{array}$ & .610 \\
\hline Logistic EuroSCORE & $5.7 \pm 6.2(204)$ & $5.5 \pm 6.1(193)$ & $8.8 \pm 7.3(11)$ & .085 \\
\hline Mechanical prosthesis & $66.7(280 / 420)$ & $66.7(264 / 396)$ & $66.7(16 / 24)$ & $>.999$ \\
\hline $\begin{array}{c}\text { Year of operation } \\
1987-1994 \\
1995-2001 \\
2002-2008 \\
2009-2015\end{array}$ & $\begin{array}{l}24.5(103 / 420) \\
23.3(98 / 420) \\
26.7(112 / 420) \\
25.5(107 / 420)\end{array}$ & $\begin{array}{l}23.7(94 / 396) \\
24.0(95 / 396) \\
26.8(106 / 396) \\
25.5(101 / 396)\end{array}$ & $\begin{array}{l}37.5(9 / 24) \\
12.5(3 / 24) \\
25.0(6 / 24) \\
25.0(6 / 24)\end{array}$ & .383 \\
\hline
\end{tabular}

Data are presented as $\%(\mathrm{n} / \mathrm{N})$ and mean \pm standard deviation or median (interquartile range). $A S$, Aortic valve stenosis; $A R$, aortic regurgitation, SAVR, surgical aortic valve replacement; $C A B G$, coronary artery bypass grafting; $P C I$, percutaneous coronary intervention; $C V A$, cerebrovascular accident; $T I A$, transient ischemic attack; $C O P D$, chronic obstructive pulmonary disease; EuroSCORE, European System for Cardiac Operative Risk Evaluation. 
TABLE 2. Baseline and procedural characteristics

\begin{tabular}{|c|c|c|c|}
\hline & Patient followed-up in Erasmus MC & Patient not followed-up in Erasmus MC & $P$ value \\
\hline Age, y & $56.9 \pm 15.5(420)$ & $66.1 \pm 11.1(1782)$ & $<.001$ \\
\hline Male sex & $66.9(281 / 420)$ & $57.4(1023 / 1782)$ & $<.001$ \\
\hline \multicolumn{4}{|l|}{ Primary indication } \\
\hline AS & $52.1(219 / 420)$ & $69.8(1243 / 1782)$ & $<.001$ \\
\hline $\mathrm{AR}$ & $25.5(107 / 420)$ & $12.7(226 / 1782)$ & $<.001$ \\
\hline Combined AS + AR & $22.4(94 / 420)$ & $17.3(308 / 1782)$ & .015 \\
\hline Bicuspid aortic valve & $24.0(101 / 420)$ & $19.2(343 / 1782)$ & .027 \\
\hline Previous cardiac operation & $28.6(120 / 420)$ & $8.6(154 / 1782)$ & $<.001$ \\
\hline SAVR & $16.7(70 / 420)$ & $4.3(76 / 1782)$ & $<.001$ \\
\hline CABG & $2.6(11 / 420)$ & $3.7(66 / 1782)$ & .276 \\
\hline Other & $9.3(39 / 420)$ & $2.4(43 / 1782)$ & $<.001$ \\
\hline Hypertension & $29.8(125 / 420)$ & $34.3(612 / 1782)$ & .073 \\
\hline Hypercholesterolemia & $12.4(52 / 420)$ & $14.8(264 / 1782)$ & .201 \\
\hline Diabetes mellitus & $9.3(39 / 420)$ & $12.2(218 / 1782)$ & .091 \\
\hline Arterial disease & $3.6(15 / 420)$ & $2.6(47 / 1782)$ & .298 \\
\hline Peripheral & $3.6(15 / 420)$ & $2.4(42 / 1782)$ & .159 \\
\hline Carotid & $0.5(2 / 420)$ & $0.3(5 / 1782)$ & .522 \\
\hline Renal failure & $2.6(11 / 420)$ & $2.3(33 / 1782)$ & .312 \\
\hline Previous myocardial infarction & $4.3(18 / 420)$ & $5.6(99 / 1782)$ & .297 \\
\hline Previous revascularization & $8.6(36 / 420)$ & $7.8(139 / 1782)$ & .599 \\
\hline Previous PCI & $6.4(27 / 420)$ & $5.1(90 / 1782)$ & .257 \\
\hline Previous CABG & $2.6(11 / 420)$ & $3.7(66 / 1782)$ & .276 \\
\hline Previous decompensated heart failure & $16.9(71 / 420)$ & $13.7(245 / 1782)$ & .097 \\
\hline \multicolumn{4}{|l|}{ Left ventricular function } \\
\hline Preserved & $77.6(287 / 370)$ & $82.5(1348 / 1633)$ & .026 \\
\hline Mildly reduced & $7.6(28 / 370)$ & $6.3(103 / 1633)$ & .376 \\
\hline Moderately reduced & $9.2(34 / 370)$ & $8.3(136 / 1633)$ & .592 \\
\hline Severely reduced & $5.7(21 / 370)$ & $2.8(46 / 1633)$ & .006 \\
\hline Atrial fibrillation & $13.3(56 / 420)$ & $13.5(241 / 1782)$ & .918 \\
\hline Previous neurologic event & $10.5(44 / 420)$ & $8.0(142 / 1782)$ & .096 \\
\hline CVA & $4.8(20 / 420)$ & $3.5(62 / 1782)$ & .212 \\
\hline TIA & $7.1(30 / 420)$ & $5.1(91 / 1782)$ & .099 \\
\hline COPD & $8.3(35 / 420)$ & $11.5(205 / 1782)$ & .061 \\
\hline Liver disease & $1.4(6 / 420)$ & $0.2(4 / 1782)$ & .001 \\
\hline History of malignancy & $8.1(34 / 420)$ & $6.1(109 / 1782)$ & .139 \\
\hline \multicolumn{4}{|l|}{ Urgency } \\
\hline Elective & $49.3(173 / 351)$ & $62.0(975 / 1573)$ & $<.001$ \\
\hline Semi-elective & $46.7(164 / 351)$ & $37.6(591 / 1573)$ & .001 \\
\hline Urgent & $4.0(14 / 351)$ & $0.4(7 / 1573)$ & $<.001$ \\
\hline Logistic EuroSCORE & $5.7 \pm 6.2(204)$ & $5.8 \pm 5.8(970)$ & .740 \\
\hline Mechanical prosthesis & $66.7(280 / 420)$ & $48.0(855 / 1782)$ & $<.001$ \\
\hline \multicolumn{4}{|l|}{ Year of operation } \\
\hline 1987-1994 & $24.5(103 / 420)$ & $16.3(290 / 1782)$ & $<.001$ \\
\hline $1995-2001$ & $23.3(98 / 420)$ & $25.4(452 / 1782)$ & .387 \\
\hline $2002-2008$ & $26.7(112 / 420)$ & $28.2(502 / 1782)$ & .536 \\
\hline 2009-2015 & $25.5(107 / 420)$ & $30.2(538 / 1782)$ & .056 \\
\hline
\end{tabular}

Data are presented as $\%(\mathrm{n} / \mathrm{N})$ and mean \pm standard deviation or median (interquartile range). AS, Aortic valve stenosis $A R$, aortic regurgitation, SAVR, surgical aortic valve replacement; $C A B G$, coronary artery bypass grafting; $P C I$, percutaneous coronary intervention; $C V A$, cerebrovascular accident; TIA, transient ischemic attack; $C O P D$, chronic obstructive pulmonary disease; EuroSCORE, European System for Cardiac Operative Risk Evaluation. 
TABLE 3. Characteristics of revascularization after SAVR

\begin{tabular}{|c|c|c|c|c|c|c|c|c|c|c|}
\hline \multirow[b]{2}{*}{ Patient } & \multirow{2}{*}{$\begin{array}{l}\text { Date of } \\
\text { SAVR }\end{array}$} & \multicolumn{5}{|c|}{ Revascularization after SAVR } & \multicolumn{2}{|c|}{$\begin{array}{c}\text { Previous } \\
\text { revascularization } \\
\text { before SAVR }\end{array}$} & \multicolumn{2}{|c|}{$\begin{array}{c}\text { Subsequent } \\
\text { revascularization(s) }\end{array}$} \\
\hline & & Date & Urgency & Lesion & Modality & Details & Date & Modality & Date & Modality \\
\hline$\# 1$ & $\begin{array}{c}\text { June } 25, \\
1987\end{array}$ & June 1, 1995 & Elective & OM, IM & PCI & & & & $\begin{array}{r}\text { September } \\
11,1995\end{array}$ & PCI \\
\hline$\# 2$ & $\begin{array}{c}\text { August 12, } \\
1987\end{array}$ & $\begin{array}{l}\text { December } \\
20,2007\end{array}$ & Elective & $\mathrm{OM}, \mathrm{PD}$ & CABG & $\begin{array}{l}\text { SVG-OM- } \\
\text { PD }\end{array}$ & & & & \\
\hline \#3 & $\begin{array}{c}\text { May 18, } \\
1988\end{array}$ & $\begin{array}{c}\text { June } 24, \\
2003\end{array}$ & Elective & LAD & CABG & SVG-LAD & & & & \\
\hline \#4 & June 3, 1988 & $\begin{array}{r}\text { November } \\
21,2003\end{array}$ & Elective & RCA & PCI & & & & & \\
\hline$\# 5$ & $\begin{array}{l}\text { September 1, } \\
1988\end{array}$ & $\begin{array}{c}\text { August 4, } \\
2015\end{array}$ & Elective & LAD, LCx & PCI & & & & & \\
\hline \#6 & $\begin{array}{c}\text { March 21, } \\
1989\end{array}$ & $\begin{array}{c}\text { November 4, } \\
1994\end{array}$ & Elective & RCA & PCI & & & & $\begin{array}{l}\text { January } 29, \\
\text { 2001, and } \\
\text { September } \\
12,2001\end{array}$ & $\begin{array}{c}\text { CABG and } \\
\text { CABG }\end{array}$ \\
\hline \#7 & July 25, 1990 & $\begin{array}{c}\text { March 29, } \\
1993\end{array}$ & Elective & LAD & CABG & LIMA-LAD & & & & \\
\hline$\# 8$ & $\begin{array}{c}\text { October 7, } \\
1993\end{array}$ & $\begin{array}{r}\text { September } \\
27,2004\end{array}$ & Elective & LAD & PCI & & & & $\begin{array}{l}\text { August 27, } \\
2012\end{array}$ & PCI \\
\hline$\# 9$ & $\begin{array}{l}\text { November 9, } \\
1993\end{array}$ & $\begin{array}{l}\text { March 10, } \\
2015\end{array}$ & Elective & LAD, RCA & PCI & & & & & \\
\hline$\# 10$ & July 1,1998 & $\begin{array}{c}\text { August } 3 \text {, } \\
2012\end{array}$ & Elective & & CABG & SVG-RCA & & & & \\
\hline$\# 11$ & $\begin{array}{c}\text { August 7, } \\
1998\end{array}$ & $\begin{array}{c}\text { June } 30 \\
2015\end{array}$ & Urgent & LAD, RCA & PCI & & & & & \\
\hline$\# 12$ & June 2, 2001 & July 4, 2014 & Elective & LAD & CABG & LIMA-LAD & & & & \\
\hline \#13 & $\begin{array}{r}\text { November } \\
28,2002\end{array}$ & $\begin{array}{l}\text { September 3, } \\
2009\end{array}$ & Elective & $\begin{array}{l}\text { LAD, IM, } \\
\text { OM }\end{array}$ & CABG & $\begin{array}{l}\text { LIMA-LAD } \\
\text { SVG-IM- } \\
\text { OM }\end{array}$ & & & & \\
\hline \#14 & $\begin{array}{c}\text { January } 31, \\
2003\end{array}$ & $\begin{array}{l}\text { December 6, } \\
2005\end{array}$ & Elective & RCA & PCI & & $\begin{array}{l}\text { October 30, } \\
2002\end{array}$ & PCI & & \\
\hline$\# 15$ & $\begin{array}{l}\text { December } \\
20,2004\end{array}$ & $\begin{array}{l}\text { October 26, } \\
2010\end{array}$ & Elective & $\mathrm{OM}$ & PCI & & & & & \\
\hline \#16 & $\begin{array}{c}\text { June } 28, \\
2006\end{array}$ & May 2, 2014 & Urgent & SVG & PCI & & May 2, 2000 & CABG & & \\
\hline \#17 & $\begin{array}{l}\text { October } 31, \\
2008\end{array}$ & $\begin{array}{c}\text { December 7, } \\
2012\end{array}$ & Elective & RCA & PCI & & $\begin{array}{c}\text { January } 19, \\
2004\end{array}$ & PCI & & \\
\hline$\# 18$ & $\begin{array}{l}\text { November } 4, \\
2008\end{array}$ & $\begin{array}{l}\text { August 27, } \\
2012\end{array}$ & Elective & RCA & PCI & & $\begin{array}{l}\text { September } \\
27,2004\end{array}$ & PCI & & \\
\hline$\# 19$ & $\begin{array}{c}\text { May 13, } \\
2009\end{array}$ & $\begin{array}{l}\text { December } \\
31,2015\end{array}$ & Elective & LAD, LCx & PCI & & $\begin{array}{c}\text { May 20, } \\
2003\end{array}$ & PCI & & \\
\hline$\# 20$ & $\begin{array}{l}\text { December 2, } \\
2011\end{array}$ & $\begin{array}{c}\text { January } 9, \\
2013\end{array}$ & Elective & $\mathrm{OM}$ & PCI & & $\begin{array}{l}\text { November 4, } \\
2011\end{array}$ & PCI & & \\
\hline \#21 & $\begin{array}{l}\text { April 27, } \\
2012\end{array}$ & $\begin{array}{c}\text { February } 5, \\
2015\end{array}$ & Urgent & LAD & PCI & & July 17, 1997 & CABG & & \\
\hline \#22 & $\begin{array}{l}\text { October } 5, \\
2012\end{array}$ & $\begin{array}{l}\text { March 11, } \\
2015\end{array}$ & Elective & LAD & CABG & LIMA-LAD & & & & \\
\hline
\end{tabular}


TABLE 3. Continued

\begin{tabular}{|c|c|c|c|c|c|c|c|c|c|c|}
\hline \multirow[b]{2}{*}{ Patient } & \multirow{2}{*}{$\begin{array}{c}\text { Date of } \\
\text { SAVR }\end{array}$} & \multicolumn{5}{|c|}{ Revascularization after SAVR } & \multicolumn{2}{|c|}{$\begin{array}{c}\text { Previous } \\
\text { revascularization } \\
\text { before SAVR } \\
\end{array}$} & \multicolumn{2}{|c|}{$\begin{array}{c}\text { Subsequent } \\
\text { revascularization(s) }\end{array}$} \\
\hline & & Date & Urgency & Lesion & Modality & Details & Date & Modality & Date & Modality \\
\hline$\# 23$ & May 2, 2013 & May 2, 2013 & Elective & PD & CABG & SVG-PD & & & & \\
\hline$\# 24$ & $\begin{array}{c}\text { October 18, } \\
2013\end{array}$ & $\begin{array}{c}\text { October 24, } \\
2013\end{array}$ & Elective & $\mathrm{LCx}$ & PCI & & & & & \\
\hline
\end{tabular}

SAVR, Surgical aortic valve replacement; $O M$, obtuse marginal artery; $I M$, intermediate artery; $P C I$, percutaneous coronary intervention; $P D$, posterior descending artery; $S V G$, saphenous vein graft; $L A D$, left anterior descending artery; $R C A$, right coronary artery; $L C x$, left circumflex artery; $C A B G$, coronary artery bypass grafting; $L I M A$, left internal mammary artery.

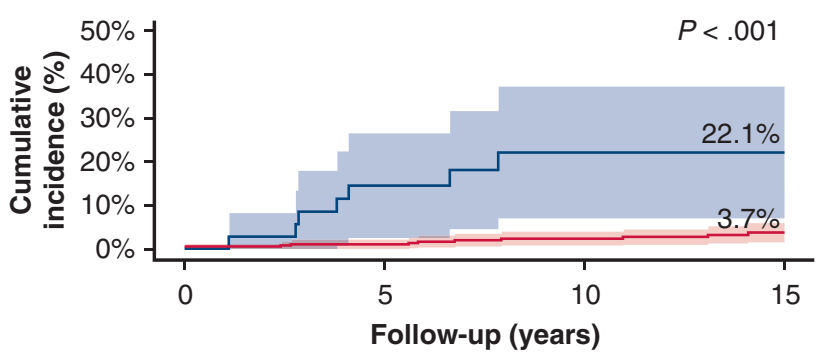

Numbers at risk

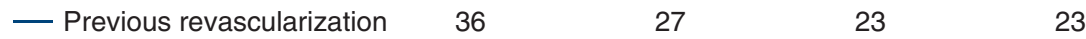

$\begin{array}{llll}\text { - No previous revascularization } & 384 & 329 & 272\end{array}$

A

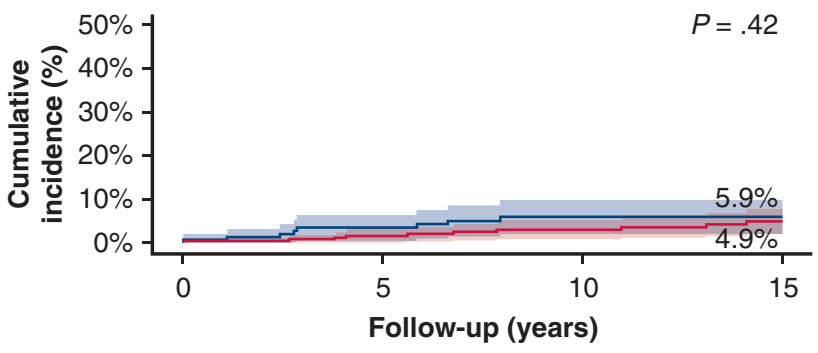

Numbers at risk

$\begin{array}{rrrrr}- \text { Age } \geq 65 & 152 & 127 & 105 & 93 \\ \text { - Age }<65 & 268 & 229 & 190 & 143\end{array}$

B

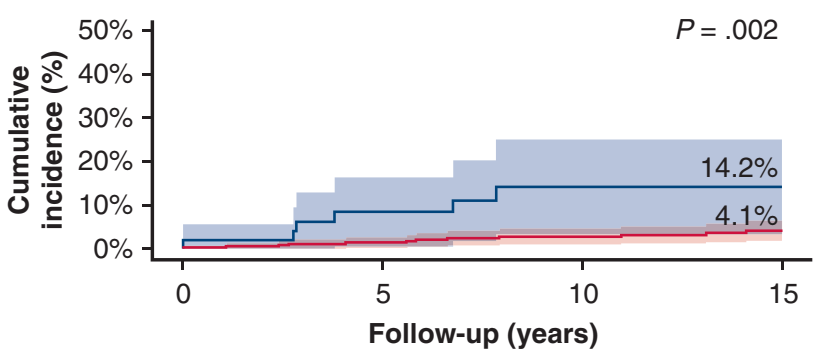

Numbers at risk

$\begin{array}{lcccc}\text { - Hypercholesterolemia } & 52 & 37 & 27 & 18 \\ \text { - No hypercholesterolemia } & 368 & 319 & 268 & 218\end{array}$

FIGURE 3. Revascularization after SAVR in various patient subgroups. Competing risk cumulative incidences of revascularization after SAVR in subgroups according to the following: (A) with and without previous revascularization. Blue line shows patients with no history of revascularization. Red line shows patients with a history of revascularization. (B) Age at SAVR younger or older than 65 years. Blue line shows patients aged 65 or older. Red line shows patients aged younger than 65 years. (C) With and without a history of hypercholesterolemia. Blue line shows patients with history of hypercholesterolemia. Red line shows patients without a history of hypercholesterolemia. 


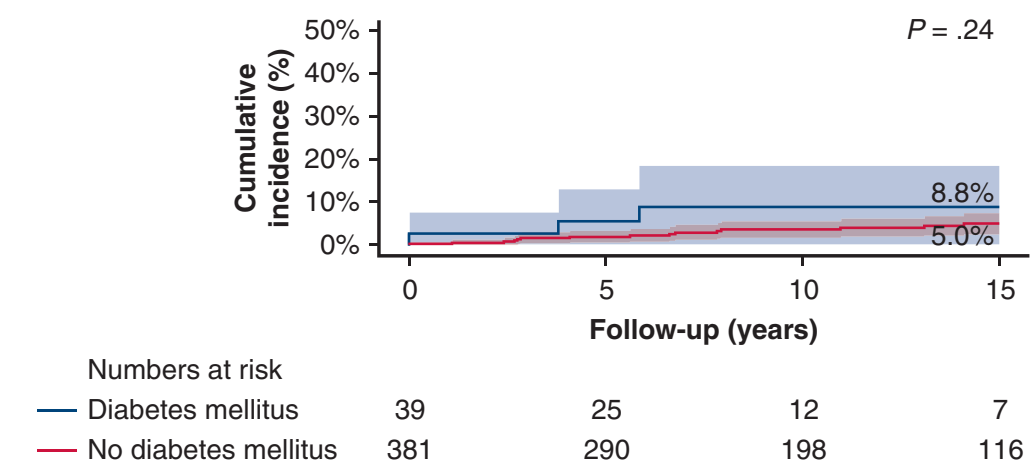

A

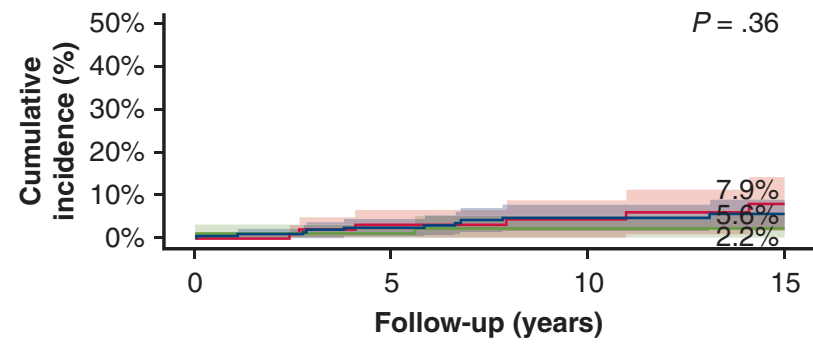

$\begin{array}{lcccc}\text { Numbers at risk } & & & & \\ \text { - AS } & 219 & 183 & 149 & 122 \\ \text { - AR } & 107 & 86 & 69 & 53 \\ \text { - Combined AS and AR } & 94 & 87 & 77 & 61\end{array}$

B

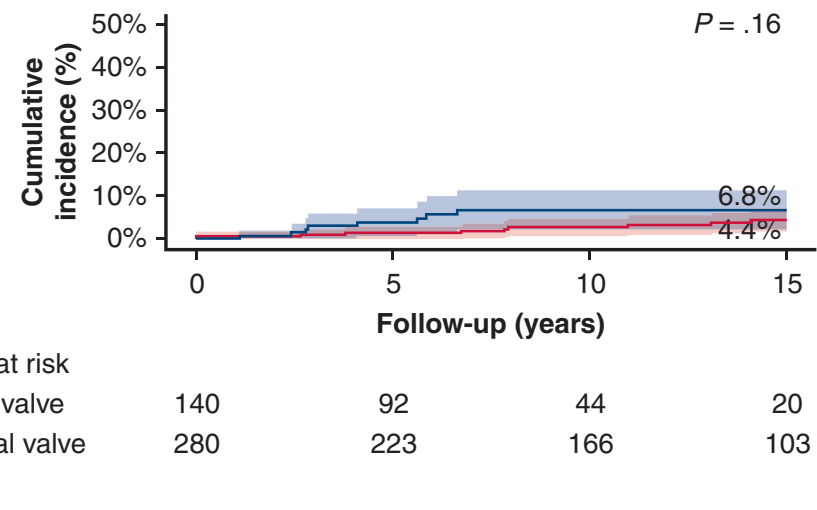

FIGURE 4. Revascularization after SAVR in various patient subgroups. Competing risk cumulative incidences of revascularization after SAVR in subgroups according to the following: (A) with and without a history of diabetes mellitus. Blue line shows patients with history of diabetes mellitus. Red line shows patients without a history of diabetes mellitus. (B) Primary indication for SAVR. Blue line shows patients undergoing SAVR for AS. Red line shows patients undergoing SAVR for AR. Green line shows patients undergoing SAVR for combined AS and AR. (C) Mechanical or biological prosthesis received. Blue line shows the use of a biological valve. Red line shows the use of a mechanical valve. $A S$, Aortic valve stenosis; $A R$, aortic regurgitation.

platform, coronary access, with potential consequences of (1) damaging the prosthetic heart valve, (2) dissecting the coronary artery, (3) acute kidney injury related to increased contrast usage, and (4) an unsuccessful procedure. ${ }^{13}$ Because CAD is present in $40 \%$ to $75 \%$ of patients undergoing TAVR, ${ }^{14}$ algorithms on obtaining coronary access have already been developed from experiences during concomitant or staged TAVR and PCI procedures. ${ }^{7}$ The presence of $\mathrm{CAD}$ in the younger population undergoing TAVR is not well known, as studies mostly consist of elderly patients. Therefore, this study is the first to systematically assess the long-term rate of revascularization after aortic valve intervention in low-risk patients without CAD. Although our population consists exclusively of 
TABLE 4. Predictors of revascularization after SAVR

\begin{tabular}{|c|c|c|}
\hline Characteristics & Univariable HR $(95 \%$ CI $) ; P$ value & Multivariable HR $(95 \%$ CI $) ; P$ value \\
\hline$\overline{\text { Age }}$ & $1.0(1.0-1.1) ; P=.16$ & \\
\hline Sex (female) & $1.5(0.6-3.4) ; P=.35$ & \\
\hline Indication AS & $1.1(0.5-2.5) ; P=.79$ & \\
\hline Indication AR & $1.1(0.4-2.7) ; P=.90$ & \\
\hline Indication AS + AR & $0.8(0.3-2.1) ; P=.68$ & \\
\hline Hypertension & $1.2(0.5-2.9) ; P=.68$ & \\
\hline Hypercholesterolemia & $5.0(2.1-11.7) ; P<.001$ & $3.4(1.3-8.6) ; P=.010$ \\
\hline Diabetes mellitus & $3.2(1.1-9.7) ; P=.037$ & $2.1(0.7-6.5) ; P=.214$ \\
\hline Arterial disease & 3.7 (0.9-15.9); $P=.08$ & \\
\hline Renal failure & $3.9(0.5-29.1) ; P=.19$ & \\
\hline Previous MI & $2.7(0.6-11.7) ; P=.17$ & \\
\hline Previous revascularization & $8.2(3.3-20.2) ; P<.001$ & $6.6(2.6-17.1) ; P<.001$ \\
\hline Decompensated heart failure & $1.8(0.7-4.6) ; P=.20$ & \\
\hline LVEF $<50 \%$ & $1.2(0.4-3.6) ; P=.76$ & \\
\hline Atrial fibrillation & $1.0(0.3-3.4) ; P=.97$ & \\
\hline Previous stroke or TIA & $0.0(0.0-18.5) ; P=.31$ & \\
\hline COPD & $1.7(0.4-7.3) ; P=.49$ & \\
\hline Urgent SAVR vs non-urgent & $1.6(0.2-12.2) ; P=.64$ & \\
\hline Log EuroSCORE & $1.1(1.0-1.1) ; P=.078$ & \\
\hline Mechanical prosthesis & $0.5(0.2-1.3) ; P=.18$ & \\
\hline
\end{tabular}

isolated SAVR procedures, it provides evidence on rates of revascularization that may be extrapolated to an overall TAVR population of low- to high-risk patients. Yet, literature also suggests that a proportion of patients might benefit from revascularization in the setting of acute coronary syndrome post-TAVR, and therefore greater incidences of revascularization could be expected in patients who initially would have been treated with medical

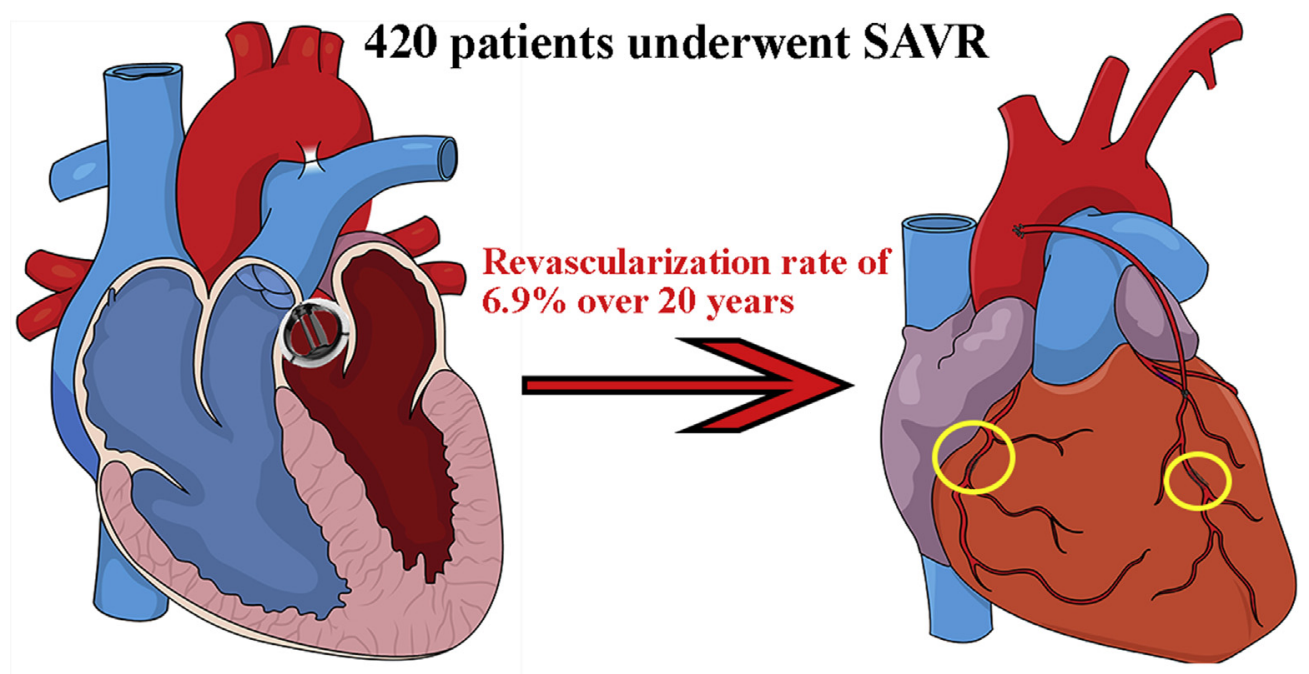

\section{Possible revascularization rate in the future younger low-risk TAVR population}

FIGURE 5. Cumulative competing risk incidence of revascularization presented as a graphical abstract. Competing risk cumulative incidence of coronary revascularization during 20-year after surgical aortic valve replacement. Coronary revascularization either done with coronary artery bypass grafting or percutaneous coronary intervention. Percutaneous coronary intervention is encircled. SAVR, Surgical aortic valve replacement; TAVR, transcatheter aortic valve replacement. 
therapy, when TAVR will expand toward the younger population. $^{15}$

Of note, the mean age of our population was 57 years old as opposed to the current TAVR population with an advanced age, but a subgroup analysis according to age showed that the long-term rate of revascularization was comparable in patients younger or older or equal to 65 years. Expanding indication to lower-risk patients may have consequences for valve choice, given the younger age, and considering that coronary access is more challenging with a supra-annular TAVR than an intra-annular TAVR.

\section{Limitations}

This is a retrospective study that has inherent shortcomings related to data collection, changes in definitions of comorbidities, and patients being lost to follow-up. However, we included only patients who were followed after SAVR at our own outpatient clinic to minimize this risk. The multivariable analyses to identify predictors of revascularization may have been underpowered due to the small number of patients that needed a revascularization procedure and the unavailability of all known risk factors for coronary artery disease. Furthermore, although the decision was made not to include patients undergoing SAVR with concomitant $\mathrm{CABG}$ in this cohort, we did not have any information on the presence and degree of nonsignificant CAD that may increase the risk of coronary revascularization during followup as a result of progression of disease.

\section{CONCLUSIONS}

In this retrospective analysis of patients who underwent isolated SAVR, the rate of requiring coronary revascularization at 20-year follow-up was relatively low. However, the rate was greater in patients who had undergone previous revascularization at the time of SAVR. These data provide some insights into requirements for coronary revascularization that may be relevant for the TAVR population. Future, larger studies are required on surgical and transcatheter cohorts to provide more insights into which patients are at particular risk of requiring coronary revascularization after aortic valve intervention.

\section{Conflict of Interest Statement}

Dr van Mieghem has received institutional research grant support from Boston Scientific, Medtronic, and Abbott and is an advisor to Medtronic and Boston Scientific. Dr Head is an employee of Medtronic, plc. Prof Kappetein is also an employee of Medtronic, plc. All other authors reported no conflicts of interest.
The Journal policy requires editors and reviewers to disclose conflicts of interest and to decline handling or reviewing manuscripts for which they may have a conflict of interest. The editors and reviewers of this article have no conflicts of interest.

\section{References}

1. Baumgartner H, Falk V, Bax JJ, De Bonis M, Hamm C, Holm PJ, et al. 2017 ESC/ EACTS Guidelines for the management of valvular heart disease. Eur Heart J. 2017;38:2739-91.

2. Nishimura RA, Otto CM, Bonow RO, Carabello BA, Erwin JP III, Fleisher LA, et al. 2017 AHA/ACC Focused Update of the 2014 AHA/ ACC Guideline for the Management of Patients With Valvular Heart Disease: a Report of the American College of Cardiology/American Heart Association Task Force on Clinical Practice Guidelines. J Am Coll Cardiol. 2017;70: 252-89.

3. Rogers T, Thourani VH, Waksman R. Transcatheter aortic valve replacement in intermediate- and low-risk patients. J Am Heart Assoc. 2018;7(10).

4. Walther T, Hamm CW, Schuler G, Berkowitsch A, Kotting J, Mangner N, et al. Perioperative results and complications in 15,964 transcatheter aortic valve replacements: prospective data from the GARY registry. J Am Coll Cardiol. 2015;65:2173-80.

5. Mack MJ, Leon MB, Thourani VH, Makkar R, Kodali SK, Russo M, et al. Transcatheter aortic-valve replacement with a balloon-expandable valve in low-risk patients. N Engl J Med. 2019;380:1695-705

6. Popma JJ, Deeb GM, Yakubov SJ, Mumtaz M, Gada H, O'Hair D, et al. Transcatheter aortic-valve replacement with a self-expanding valve in low-risk patients. N Engl J Med. 2019;380:1706-15.

7. Yudi MB, Sharma SK, Tang GHL, Kini A. Coronary angiography and percutaneous coronary intervention after transcatheter aortic valve replacement. J Am Coll Cardiol. 2018;71:1360-78.

8. Abdel-Qadir H, Fang J, Lee DS, Tu JV, Amir E, Austin PC, et al. Importance of considering competing risks in time-to-event analyses: application to stroke risk in a retrospective cohort study of elderly patients with atrial fibrillation. Circ Cardiovasc Qual Outcomes. 2018;11:e004580.

9. Satagopan JM, Ben-Porat L, Berwick M, Robson M, Kutler D, Auerbach AD. A note on competing risks in survival data analysis. Br J Cancer. 2004;91: 1229-35.

10. Scrucca L, Santucci A, Aversa F. Competing risk analysis using R: an easy guide for clinicians. Bone Marrow Transplant. 2007;40:381-7.

11. Scrucca L, Santucci A, Aversa F. Regression modeling of competing risk using R: an in-depth guide for clinicians. Bone Marrow Transplant. 2010;45: 1388-95.

12. Neumann FJ, Sousa-Uva M, Ahlsson A, Alfonso F, Banning AP, Benedetto U, et al. 2018 ESC/EACTS Guidelines on myocardial revascularization. Eur Heart J. 2019;40:87-165.

13. Alqahtani F, Ziada K, Rihal CS, Alkhouli M. Incidence and outcomes of early percutaneous coronary intervention after isolated valve surgery. Catheter Cardiovasc Interv. 2019;93:583-9.

14. Goel SS, Ige M, Tuzcu EM, Ellis SG, Stewart WJ, Svensson LG, et al. Severe aortic stenosis and coronary artery disease-implications for management in the transcatheter aortic valve replacement era: a comprehensive review. J Am Coll Cardiol. 2013;62:1-10.

15. Mentias A, Desai MY, Saad M, Horwitz PA, Rossen JD, Panaich S, et al. Incidence and outcomes of acute coronary syndrome after transcatheter aortic valve replacement. JACC Cardiovasc Interv. 2020;13:938-50.

Key Words: aortic stenosis, aortic valve replacement, transcatheter, coronary artery bypass grafting, percutaneous coronary intervention 